Competencias TIC para la gestión del conocimiento: un aporte desde el Modelo TPACK

Candy Padrón y María de la Soledad Bravo ( pp. 49-73)
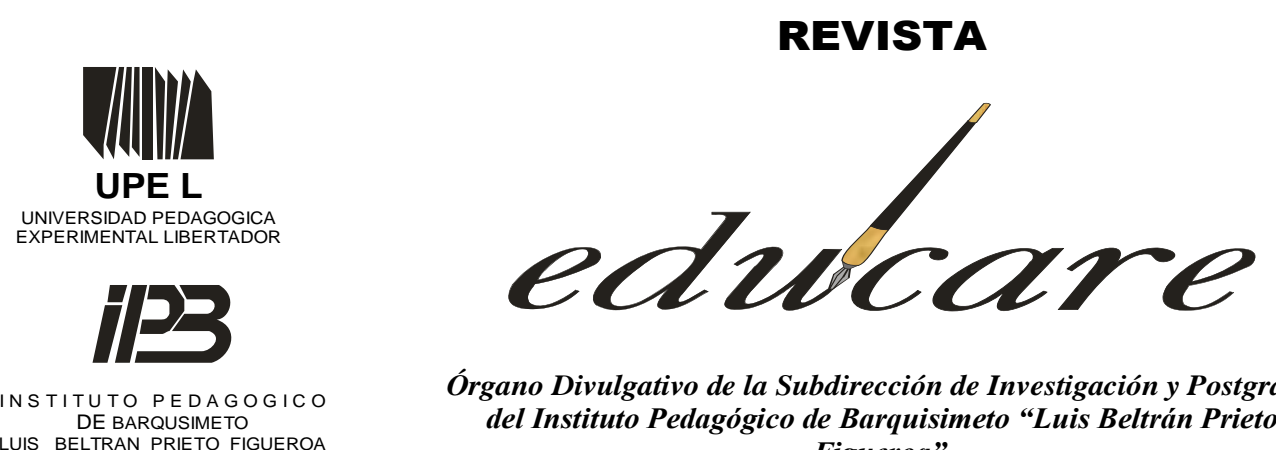

Órgano Divulgativo de la Subdirección de Investigación y Postgrado del Instituto Pedagógico de Barquisimeto "Luis Beltrán Prieto Figueroa"

BARQUISIMETO - EDO. LARA - VENEZUELA

NUEVA ETAPA

FORMATO ELECTRÒNICO

DEPOSITO LEGAL: ppi201002LA3674

Volumen 18 № 3

ISSN: 2244-7296

\title{
COMPETENCIAS TIC PARA LA GESTIÓN DEL CONOCIMIENTO: UN APORTE DESDE EL MODELO TPACK
}

ICT SKILLS FOR KNOWLEDGE MANAGEMENT:

A CONTRIBUTION FORM THE MODEL TPACK

\begin{abstract}
Candy Padrón*
María de la Soledad Bravo*

*Universidad Pedagógica Experimental Libertador - Instituto Pedagógico de Barquisimeto (UPEL - IPB)
\end{abstract}




\section{COMPETENCIAS TIC PARA LA GESTIÓN DEL CONOCIMIENTO:} UN APORTE DESDE EL MODELO TPACK

\section{ICT SKILLS FOR KNOWLEDGE MANAGEMENT: A CONTRIBUTION FORM THE MODEL TPACK}

REVISIÓN DOCUMENTAL

Recibido: 01-10-2014
Candy Padrón*

María Bravo**

UPEL - IPB

Aceptado: 15-12-2014

\section{RESUMEN}

En el ámbito educativo, la intención de integrar las Tecnologías de Información y Comunicación (TIC) ha sido mejorar los procesos de enseñanza, aprendizaje y gestión. Para realizar estos procesos de manera exitosa, se requiere el desarrollo de una serie de competencias que trasciendan el uso instrumental de la tecnología para ir hacia usos pedagógicos, investigativos y gerenciales. En tal sentido, la presente investigación documental, tiene como propósito ofrecer a los docentes en formación y ejercicio orientaciones teóricas para abordar la incorporación de las TIC en los ámbitos referidos a partir de las competencias mínimas necesarias y a la luz del modelo TPACK cuyo nombre viene dado por las siglas en inglés Technological Pedagogical And Content Knowledge, que contempla tres conocimientos fundamentales para abordar la integración tecnológica curricular: el conocimiento pedagógico, tecnológico y disciplinar. Se estima que su empleo favorece procesos de implantación de las TIC de manera contextualizada.

Descriptores: Formación docente, competencias TIC, gestión del conocimiento.
In education, the intention to integrate Information and Communication Technologies (ICT) has been to improve the teaching, learning and management. To execute these processes successfully, developing a set of skills that go beyond the instrumental use of technology to go into teaching, research and management applications is required. In that sense, this documentary research aims to provide teachers in training and exercise theoretical orientations to address the incorporation of ICT in the areas referred from the minimum skills required in the light TPACK, model whose name is given by the acronym and Technological, Pedagogical and Content knowledge, which provides basic knowledge to address integration technology curriculum: pedagogical, technological and disciplinary knowledge. It is estimated that its use promotes processes of implementation of contextualized ICT.

Keywords: teaching training, ICT skills, management knowledge.

\footnotetext{
*Magister en Profesora de Inglés. Magister en Gerencia Educacional. Docente del Departamento de Formación Docente. Correo Electrónico: candypad@gmail.com

**Doctora en Ciencias de la Educación. Magister en Educación Superior. Magister en Tecnología y Diseño Educativo. Profesor de Educación Industrial Mención Mecánica. Profesora Asociado-Departamento de Formación Docente UPEL-IPB. Coordinadora del Núcleo de Investigación Docencia, Innovación y Tecnología. Correo Electrónico: mariasoledadbravob@gmail.com
}

Revista EDUCARE, Volumen 18, Número 3, Septiembre - Diciembre 2014. ISSN: 2244-7296 Página 50 


\section{Competencias TIC para la gestión del conocimiento: un aporte desde el Modelo TPACK \\ Candy Padrón y María de la Soledad Bravo ( pp. 49-73)}

\section{INTRODUCCIÓN}

Las TIC se han convertido en una herramienta insustituible tanto en las actividades profesionales como personales de los últimos 20 años, por lo que desarrollar competencias para su uso sigue siendo un factor de éxito en la formación académica de diversas carreras para pasar de una sociedad de la información a una sociedad del conocimiento. En el ámbito docente, estas competencias se diversifican a partir de la necesidad de crear nuevos espacios de aprendizaje, nuevos medios y nuevas formas de interacción para responder a la necesidad de formación de manera asíncrona, deslocalizada y permanente, que demanda la sociedad del conocimiento.

Ante la posibilidad de caer en posturas tecnocéntricas que conduzcan a creer que las TIC per se permitirán superar todos los problemas educativos, se requiere consolidar un pensamiento reflexivo en torno a su uso y desarrollar competencias de diversa índole, que conduzcan a fortalecer al profesional de la docencia en su hacer tecnológico, comunicativo, de gestión e investigación, de modo que posea un abanico de habilidades y saberes que le permitan afrontar las necesidades de inclusión e innovación con las TIC en el nuevo escenario educativo. Ya que, al presentar a los docentes las TIC bajo modelos de integración complejos en la cual necesitan ardua especialización al respecto, la incorporación al ámbito educativo se manifiesta tardío, pues se deja a un lado la relevancia del fin último de la educación y se piensa que son aquellas sobre las cuales se deben especializar. Es por esto que algunas veces la incorporación de tecnologías al currículo se hace débil y genera un rezago en las comunidades académicas en cuanto al manejo de estas herramientas.

En tal sentido, el trabajo que se presenta es de naturaleza documental y se apoya en la revisión y análisis de fuentes impresas y electrónicas en el campo de las TIC aplicadas a la educación, por lo que el propósito que nos guía es presentar una serie de aspectos teóricos vinculados con las competencias que debe poseer y/o desarrollar el docente a partir de lo que contemplan autores y organizaciones reconocidas, así como la experiencia de las autoras tanto en el ámbito de las TIC como de la formación docente. Además, se incorporan

Revista EDUCARE, Volumen 18, Número 3, Septiembre - Diciembre 2014. ISSN: 2244-7296 Página 51 


\section{Competencias TIC para la gestión del conocimiento: un aporte desde el Modelo TPACK}

Candy Padrón y María de la Soledad Bravo ( pp. 49-73)

los conocimientos necesarios para la gestión del conocimiento, desde el punto de vista del Modelo TPACK en las instituciones educativas.

\section{DE LA SOCIEDAD DE LA INFORMACIÓN A LA SOCIEDAD DEL CONOCIMIENTO UN PROCESO VEHICULADO POR LAS TIC}

Las TIC, han surgido por la integración de las bondades de la informática y la telemática, revitalizando los procesos de generación, transformación, almacenamiento, distribución e intercambio de todo tipo información en tiempo real mediante la digitalización en diversos formatos. Esta digitalización ha permitido la integración de los medios como una de las características distintivas de las TIC, junto con otras como interactividad, innovación, elevados parámetros de calidad de imagen y sonido, mayor influencia sobre los procesos que sobre los productos, automatización, interconexión y diversidad. Azinian (2009).

Así, la sociedad de la información se ha redimensionado a partir del uso intensivo de internet, ya que la información se transmite de manera más rápida sin importar fronteras de tiempo y espacio, al respecto, García, Ruíz y García (2009) definen la sociedad de la información como un sistema social, basado en un soporte tecnológico, que facilita el acceso a todo tipo de información, de datos, independientemente de las coordenadas tempogeográficas en que estén insertos. No obstante, clarifican que la información es importante, pero aún más importante es enseñar destrezas, procedimientos y habilidades para acceder al mundo de la información, generando así un auténtico y personal conocimiento.

Esta realidad genera un nuevo reto para las sociedades en el campo laboral, el cual viene a representar un factor de competitividad, el conocimiento generado a través de la información y se convierte en el nuevo capital para la globalización. Por ello la UNESCO (2010) resalta que la sociedad dejó de ser sólo mano de obra, para ser una sociedad alta en conocimiento. Los trabajos que realizarán los futuros estudiantes no podemos ni imaginarlos, aprenden de manera distinta y eso torna posible re-examinar tanto los

Revista EDUCARE, Volumen 18, Número 3, Septiembre - Diciembre 2014. ISSN: 2244-7296 Página 52 


\section{Competencias TIC para la gestión del conocimiento: un aporte desde el Modelo TPACK}

\section{Candy Padrón y María de la Soledad Bravo ( pp. 49-73)}

contenidos como la forma de enseñarlos.

En tal sentido, como consecuencia de la sociedad de la información surge la sociedad del conocimiento, dado que ya no es primordial la información per se, sino como ésta es procesada y aprovechada por aquellos quienes están conscientes de que el verdadero valor radica en la apropiación de la misma tanto para la sociedad como para el individuo. Respecto a la sociedad del conocimiento Krüger (2006) expresa que:

El concepto actual de la 'sociedad del conocimiento' no está centrado en el progreso tecnológico, sino que se considera como un factor del cambio social, entre otros, como por ejemplo, la expansión de la educación. Según este enfoque, el conocimiento será cada vez más la base de los procesos sociales en diversos ámbitos funcionales de las sociedades. Crece la importancia del conocimiento como recurso económico, lo que conlleva la necesidad de aprender a lo largo de toda la vida. (p.7)

En virtud de lo expuesto, debemos reconocer que a pesar de que las TIC son importantes, lo más imprescindible es manejar el conocimiento. Así pues, una institución educativa puede tener un equipamiento óptimo en TIC, pero si el cuerpo docente no posee un nivel básico de conocimiento para su utilización, estas serán subutilizadas. Asimismo, los docentes, sobre todo los del nivel universitario, deben propiciar en sus estudiantes, además de la capacitación en su especialidad, la facultad de poder continuar aún después del pregrado en una apropiación continua de la información convirtiéndose en un proceso constante transformador del conocimiento. A continuación se destacan características esenciales de cada una de estas sociedades.

\section{Cuadro 1}

Características de la sociedad de la información y la sociedad del conocimiento.

\begin{tabular}{llll}
\hline \multicolumn{2}{l}{ Sociedad de la información } & \multicolumn{2}{c}{ Sociedad del conocimiento } \\
\hline - & Convergen los soportes & Convergen los contenidos \\
- & La información es unívoca, & - & La información se reelabora, se \\
& unidireccional & & interpreta, se comunica \\
- & Predomina el sistema & - & Predomina el sujeto \\
- & Masiva, indiscriminada & - & Individual, diferenciadora \\
- Entrada en los contenidos & - & Entrada en el proceso \\
- Democrática & - & Participativa \\
\hline
\end{tabular}

Tomado de García, Ruíz y García (ob. cit)

Revista EDUCARE, Volumen 18, Número 3, Septiembre - Diciembre 2014. ISSN: 2244-7296 Página 53 


\section{Competencias TIC para la gestión del conocimiento: un aporte desde el Modelo TPACK}

\section{Candy Padrón y María de la Soledad Bravo ( pp. 49-73)}

Como puede observarse, la sociedad del conocimiento busca esa apropiación y transformación de la información y es allí donde el papel del docente es de gran relevancia, pues en las universidades, más que la formación en una disciplina específica, se debe brindar a los estudiantes herramientas que les permitan estar inmersos en la sociedad actual y ser competitivos en el mercado laboral. Este nuevo escenario implica volver la mirada hacia las instituciones formadoras de docentes, de modo que sus egresados posean las competencias necesarias para llevar a cabo el proceso de integración de las TIC al currículo, pues que no basta con incorporar tecnologías en las aulas, es necesario hacer un uso pertinente de las mismas y convertirlas en herramientas de apoyo para la construcción de saberes.

Cabe destacar que en el ámbito nacional se han implementado medidas de equipamiento tecnológico en diversas instituciones, sin embargo, la capacitación de los docentes no ha marchado a la par, por lo que el aprovechamiento de dichas tecnologías no ha sido óptimo. Al respecto, Ostériz (2012) comenta que:

En los últimos años, solamente algunos gobiernos empezaron en forma no muy inteligente, entregando computadores a algunos, sin preparar a los docentes, sin diseñar planes educativos para el E3 (entorno de tecnologías avanzadas), y olvidándose de que a los que hay que preparar, en primer lugar, es a los docentes de todos los niveles que estén dispuestos a llevar a cabo el proyecto de cambio educativo. (p. 3)

De manera que en Venezuela, a pesar de que en sectores como la banca, la investigación científica, el comercio electrónico, espectáculos, salud, entre otros, se han adaptado en la medida del auge de las tecnologías, se observa, un rezago del sector educativo en el proceso de adaptación al nuevo entorno social. Por lo tanto, se requiere profundizar en la revisión y aplicación de modelos que faciliten el proceso de transición de la educación tradicional hacia una educación apoyada en las TIC a partir de la gestión del conocimiento.

\section{Hacia la Gestión del Conocimiento}

Posterior al surgimiento de la sociedad del conocimiento, las organizaciones 


\section{Competencias TIC para la gestión del conocimiento: un aporte desde el Modelo TPACK}

\section{Candy Padrón y María de la Soledad Bravo ( pp. 49-73)}

comenzaron a notar que este era su capital más valioso. Tener en la organización personal capaz de utilizar de forma proactiva su conocimiento proporciona herramientas útiles que brindan oportunidades a la hora de resolver situaciones problemáticas. Por consiguiente, las empresas prestan gran atención al trabajo de conocimiento, el cual para Flores y Torres (2011), es aquel que requiere que una persona recabe, procese, modifique y organice información para generar soluciones diferentes a problemas específicos que se presentan en un contexto determinado. Al notar que el trabajo del conocimiento permite adaptarse a situaciones específicas, el ámbito educativo enfoca su atención a la gestión del mismo, a pesar de que esto ya se realizaba de manera empírica.

Ante lo expuesto, el trabajo del conocimiento se relaciona con la labor docente y la necesidad de emprenderla con un enfoque gerencial. Esto le permite a los docentes no solo obtener conocimiento, sino compartirlo para obtener una gestión de él que aporte a las instituciones educativas un máximo aprovechamiento del capital intelectual con el que cuenta, con beneficios no solo para la institución educativa, sino además para la comunidad y la sociedad misma. Con respecto a esto, Flores y Torres (ob. cit.) recalcan que al momento de reconceptualizar la práctica docente como "trabajo de conocimiento," se abre una posible explicación para entender por qué, a pesar de contar cada vez con mayor conocimiento sobre la enseñanza y el aprendizaje, y mayor preparación y recursos para los maestros, los resultados del trabajo docente no son los esperados, y cómo resolver la aparente paradoja de entre más sabemos sobre cómo educar, peor lo hacemos.

Adicionalmente, señalan que el profesor, por un lado, constituye parte del capital humano de una sociedad de conocimiento y por otro, es un elemento clave en el desarrollo del capital humano de la sociedad de conocimiento por la esencia de su función; y apuntan que "el docente debe lograr identificar cuales conocimientos son indispensables para el ejercicio de la práctica reflexiva, tomar decisiones conscientes y racionales durante su práctica y además interesarse en la justicia social y ética de la educación” (p. 190).

De tal forma, la labor docente no debe ser vista solo como trabajo de conocimiento, sino que debería basarse en una teoría gerencial. Fontcuberta (citado en Azinian, ob. cit) explica que la gestión de conocimiento significa, en principio, saber cómo acceder a las

Revista EDUCARE, Volumen 18, Número 3, Septiembre - Diciembre 2014. ISSN: 2244-7296 Página 55 


\section{Competencias TIC para la gestión del conocimiento: un aporte desde el Modelo TPACK}

Candy Padrón y María de la Soledad Bravo ( pp. 49-73)

informaciones, seleccionarlas, articularlas y aplicarlas a un determinado objetivo. Se habla entonces de ir más allá, e introducir la teoría de Gestión de Conocimiento en dicho ámbito, para dar respuesta a la paradoja antes mencionada, que el docente no trabaje solamente de forma individual, sino que también tenga la oportunidad de trabajar en conjunto en la búsqueda de nuevas estrategias, todo esto con el fin de obtener un mejor ejercicio de la docencia.

Por lo tanto, el sector educativo y en especial el universitario, requiere mejorar la gestión del conocimiento para hacerlo útil y pertinente. Sin embargo, para lograr que los docentes se mantengan en un aprendizaje continuo en pro de una mejor gestión de conocimiento, es necesaria la práctica reflexiva, en la cual cada docente tome consciencia de la importancia, para él y sus estudiantes, de mantenerse actualizado con sus conocimientos y compartirlos con otros para crear nuevas formas de aprendizaje.

Considerando elementos como la práctica reflexiva y el uso de tecnologías para apoyo de la gestión de conocimiento, Azinian (ob. cit.) asegura que solo un docente capacitado y formado en la reflexión puede tomar las decisiones apropiadas para el rediseño de los entornos de aprendizaje que requiere la integración curricular de las TIC, con el fin de producir una transformación cualitativa de las prácticas pedagógicas. Por consiguiente, no se puede realizar una óptima gestión de conocimiento sin tomar en cuenta la importancia que presentan los recursos tecnológicos utilizados bajo una perspectiva reflexiva, esto implica que el docente desarrolle competencias específicas para el nuevo entorno social y formativo.

\section{Competencias TIC y Formación Docente}

Las TIC han pasado a formar parte relevante en el proceso educativo, la sociedad de la información y del conocimiento han motivado el uso de las tecnologías en todos los sectores ya que podemos acceder a la información a través de múltiples medios y a partir de diferentes herramientas tecnológicas, por lo que las universidades tienen el deber de realizar transformaciones para adaptarse a las nuevas necesidades de la sociedad actual y sus docentes requieren asumir el reto de incorporar las TIC tanto en los procesos

Revista EDUCARE, Volumen 18, Número 3, Septiembre - Diciembre 2014. ISSN: 2244-7296 Página 56 


\section{Competencias TIC para la gestión del conocimiento: un aporte desde el Modelo TPACK}

\section{Candy Padrón y María de la Soledad Bravo ( pp. 49-73)}

académicos como gerenciales.

Al respecto, Melaré (2007) señala que:

Las competencias necesarias para el trabajo con las tecnologías trascienden los conocimientos clásicos y las formas de aprendizaje de la educación formal. Por eso, la necesidad de que sean orientadas en la formación de profesores, para que ellos estén en condiciones de utilizarlas efectivamente como herramientas pedagógicas en su acción docente. (p.28)

Asimismo, el docente debería buscar del dominio de las competencias tecnológicas, basadas en modelos que ayuden a su integración. Sin embargo, a pesar de que las TIC se han venido incorporando a la educación desde hace algunos años, "algunos profesores se sienten inseguros ante esta invasión en su tradicional ámbito de trabajo, teniendo que convivir con ellas sin haber sido convenientemente preparados, lo que ha proporcionado inicialmente actitudes de rechazo hacia su utilización" (Castillo y Cabrerizo, 2006, p. 41).

Diaz Barriga (2010), acota que si la meta de la educación en competencias se ubica en el plano de la formación por competencias de los docentes para el empleo de las TIC, hay que puntualizar que esta no puede quedarse solo en la formación sino que debe enfocarse en su aplicación y uso. De esta manera, un docente competente en el empleo de las TIC será aquel que sepa qué, cómo y cuándo enfrentar las situaciones que le plantea su acción educativa, siendo capaz de reaccionar ante la complejidad y la incertidumbre de la cotidianidad del aula. Además apunta que cuando se adopta la idea de competencia como prescripción abierta, se concibe que un docente competente es aquel que sabe tomar iniciativas y decisiones, negociar, hacer elecciones en condiciones de riesgo, innovar y asumir responsabilidades. Y agrega:

Para enseñar competencias (y para formar a los docentes en competencias con la meta de que transformen las prácticas en el aula), no basta con elaborar referenciales de competencias e insertarlas en el currículo, tampoco con la transmisión de conocimientos o la automatización de procedimientos. Para enseñar competencias se requiere crear situaciones didácticas que permitan enfrentar directamente a los estudiantes o a los docentes en formación y/o servicio a las tareas que se espera que resuelvan. Se requiere asimismo que adquieran y aprendan a movilizar los recursos indispensables y que lo hagan con fundamento en procesos de reflexión metacognitiva o autorregulación.

Revista EDUCARE, Volumen 18, Número 3, Septiembre - Diciembre 2014. ISSN: 2244-7296 Página 57 


\section{Competencias TIC para la gestión del conocimiento: un aporte desde el Modelo TPACK}

Candy Padrón y María de la Soledad Bravo ( pp. 49-73)

Los programas y objetivos de formación, desde la mirada de una educación por competencias, no se derivan en términos de conocimientos estáticos o declarativos, sino en términos de actividades generativas y tareas-problema que la persona en formación deberá enfrentar, de modo que se generen situaciones propias de la especialidad del participante, escenarios similares a su campo de acción, estudios de caso, entre otras actividades significativas y relevantes.

Por otra parte, las competencias son mucho más que un saber hacer en un contexto, pues van más allá del plano de la actuación e implican compromiso, disposición a hacer las cosas con calidad, raciocinio, manejo de una fundamentación conceptual y comprensión; por lo tanto, contemplan tres dimensiones: La afectivo motivacional; la cognitiva y la actuacional. Además, son procesos complejos porque implican la interacción con muchas dimensiones del ser humano y del contexto, como también la asunción y afrontamiento de la incertidumbre, uno de los grandes retos para la educación (Morin en Tobón, Rial Sánchez, Carretero y García, 2006).

En correspondencia con los autores precitados, Henríquez, Veracoechea y Gómez de Ugel (2011), coinciden en manifestar que se entiende por competencia la combinación de tres elementos o saberes, los cuales permitirán a un individuo desarrollarse eficiente y eficazmente dentro del área profesional (a) conocimientos o saber conocer, se identifican las diferentes fuentes de información, acorde con sus potencialidades y servicios; (b) destrezas o saber hacer, se sistematiza la información para emplearla en la realización de tareas de acuerdo con el tipo de actividad y (c) actitudes o saber ser, demuestra interés en la comprensión del conocimiento, que se evidencia en sus reflexiones.

Aun cuando existen diversos documentos en torno a las competencias TIC para docentes, asumimos, en correspondencia con la postura de Corredor (2014), que definir un marco referencial de competencias no puede garantizar una representación consensuada, completa y estable de una profesión. Un referencial de competencias es un instrumento para pensar las prácticas, debatir sobre la profesión, descubrir los aspectos emergentes o las zonas controvertidas, por ello incluimos en este artículo, las que en nuestra opinión como docentes investigadoras pueden aplicarse al ámbito educativo y particularmente en las

Revista EDUCARE, Volumen 18, Número 3, Septiembre - Diciembre 2014. ISSN: 2244-7296 Página 58 


\section{Competencias TIC para la gestión del conocimiento: un aporte desde el Modelo TPACK \\ Candy Padrón y María de la Soledad Bravo ( pp. 49-73)}

universidades formadoras de docentes, ya que el tema de las competencias TIC, ha sido desde hace más de una década preocupación de investigadores y organizaciones como la UNESCO, Ministerios de Educación, entre otros.

Al respecto, Marcelo y otros (2002), presentaban una amplia gama de competencias al organizarlas en tres áreas: la tecnológica, la didáctica y la tutorial, las cuales no han perdido vigencia.

La competencia tecnológica, la relacionaban con el dominio de destrezas técnicas básicas, (hardware, gestión de ficheros, navegación, diseño de aplicaciones multimedia, páginas web, software de autor, aplicaciones de Internet), interés por la renovación y actualización permanente y capacidad para simplificar los aspectos tecnológicos y procedimentales para que el estudiante pueda centrarse en lo exclusivamente formativo.

La competencia didáctica, la vinculaban con el conocimiento de las teorías del aprendizaje y los principios del aprendizaje adulto, dominio científico del área específica de conocimiento, capacidad de adaptación a nuevos formatos de formación, actitud creativa e innovadora ante las múltiples oportunidades que ofrece la red, dotes para la comunicación y transmisión para seleccionar y organizar contenidos de manera significativa, capacidad para el diseño de ambientes de aprendizaje basados en la red y de posible autodirección y autorregulación por parte del estudiante y capacidad para crear materiales y tareas relevantes y de fácil transferencia a situaciones específicas.

Finalmente, la competencia tutorial, se refería a habilidades de comunicación, capacidad de adaptación a los distintos usuarios, orientación realista de la planificación, mentalidad abierta y constancia para realizar seguimiento del progreso del alumno y predisposición a asumir roles polivalentes de acuerdo a las situaciones de aprendizaje.

Al tema de las competencias docentes se le ha asignado tanta importancia a nivel internacional, que la UNESCO (2008) publicó sus nomas sobre Competencias en TIC para docentes, en las cuales ofrece "orientaciones destinadas a todos los docentes y más concretamente directrices para planear programas de formación del profesorado y ofertas de cursos que permitirán preparar a los docentes para desempeñar un papel esencial en la capacitación tecnológica de los alumnos". (p. 3). Estas normas forman parte de un primer

Revista EDUCARE, Volumen 18, Número 3, Septiembre - Diciembre 2014. ISSN: 2244-7296 Página 59 


\section{Competencias TIC para la gestión del conocimiento: un aporte desde el Modelo TPACK}

\section{Candy Padrón y María de la Soledad Bravo ( pp. 49-73)}

proyecto que se desarrolla en tres documentos: (a) el primero trata del Marco de políticas; (b) el segundo examina los componentes de la reforma de la educación y presenta un marco de conjuntos de competencias que corresponde a los distintos enfoques en materia de políticas y los componentes del sistema educativo y (c) el tercero proporciona una descripción detallada de las competencias específicas que los docentes deben adquirir en el contexto de cada conjunto de competencias o módulo, el segundo proyecto estará orientado a crear un mecanismo destinado a aprobar los programas de formación que sean conformes a las Normas UNESCO.

Según la UNESCO (ob. cit), las TIC exigen que los docentes desempeñen nuevas funciones, y también requieren nuevas pedagogías y nuevos planteamientos de la formación de docentes, por lo que lograr integrarlas en el aula dependerá de su capacidad para estructurar el entorno de aprendizaje de forma no tradicional, fusionar las tecnologías con nuevas pedagogías y fomentar clases dinámicas en el plano social, estimulando la interacción cooperativa, el aprendizaje en colaboración y el trabajo de grupo. Esto exige adquirir un conjunto diferente de competencias de gestión de la clase. En tal sentido apunta que en el futuro las competencias fundamentales comprenderán la capacidad para desarrollar métodos innovadores de utilización de la tecnología con vistas a mejorar el entorno del aprendizaje y la capacidad para estimular la adquisición de nociones básicas de tecnología, la profundización de los conocimientos y la creación de éstos.

En opinión de esta organización, la formación profesional del docente será un componente fundamental de esta mejora de la educación. No obstante, el perfeccionamiento profesional del docente sólo tendrá un impacto si se centra en cambios específicos del comportamiento del docente en clase $\mathrm{y}$, en particular, si ese perfeccionamiento es permanente y se armoniza con otros cambios en el sistema educativo. Por consiguiente, el proyecto relativo a las Normas UNESCO sobre Competencias en TIC para Docentes interpreta las repercusiones que cada uno de los tres enfoques de mejora de la educación tienen en los cambios de cada uno de los componentes del sistema educativo: política, plan de estudios y evaluación, pedagogía, utilización de la tecnología, organización y administración de la escuela, y perfeccionamiento profesional del docente.

Revista EDUCARE, Volumen 18, Número 3, Septiembre - Diciembre 2014. ISSN: 2244-7296 Página 60 
En el gráfico 1 se muestran los tres enfoques propuestos (1) Nociones básicas de las TIC; (2) Profundización del conocimiento y (3) Generación del Conocimiento y las competencias generales esperadas en cada enfoque, en las normas de los Módulos UNESCO sobre competencias TIC de los docentes, se detalla las competencias específicas. Se sugiere revisar el documento: Normas UNESCO Enfoques y competencias TIC para docentes (NUECTIC) de UNESCO (ob. cit).

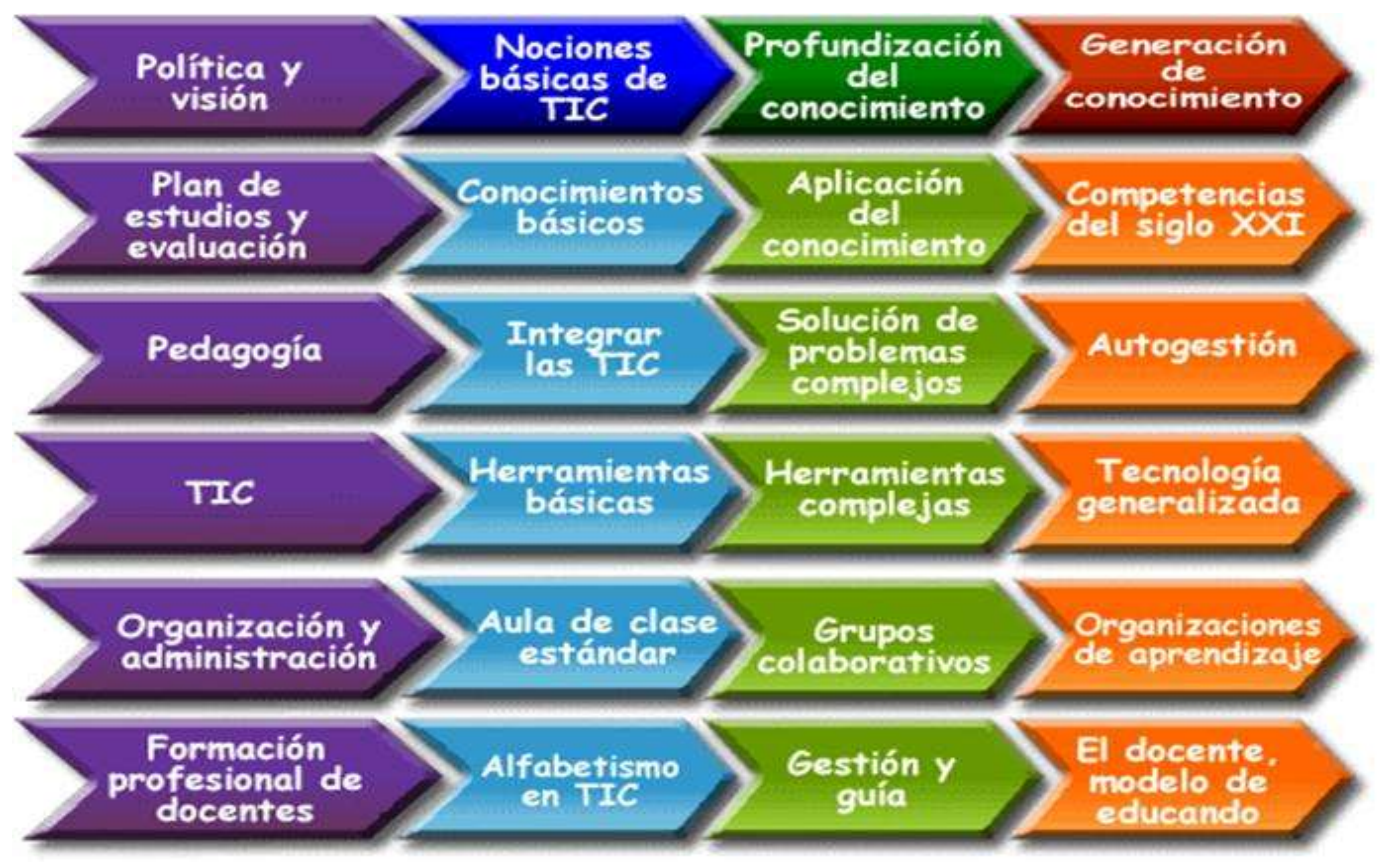

Gráfico 1.Normas UNESCO Enfoques y competencias TIC para docentes

Por su parte Mauri y Onrubia (2008), resaltan el rol del docente como e-mediador, entendido como alguien que proporciona ayudas educativas ajustadas a la actividad constructiva del alumno empleando para ello las TIC, diferenciando entre la interactividad tecnológica y la interactividad pedagógica y entre el plano de diseño y el del desarrollo de la enseñanza, tomando en cuenta la naturaleza constructiva, social y comunicativa de la mediación. Integrando los aportes de Berge, hepp, McPerson y Nunes, Salmon, Monereo y Savery (citados por Mauri y Onrubia, ob. cit) quienes concretan el rol de e-mediador en cuatro grandes ámbitos: el pedagógico, relacionado con el desarrollo de un proceso de aprendizaje virtual eficaz; el social, vinculado al desarrollo de un entorno de aprendizaje 


\section{Competencias TIC para la gestión del conocimiento: un aporte desde el Modelo TPACK \\ Candy Padrón y María de la Soledad Bravo ( pp. 49-73)}

con un clima emocional y afectivo confortable en el que los alumnos sienten que el aprendizaje es posible; el de organización y gestión, relacionado con el establecimiento de un diseño instruccional adecuado que incluye animar a los implicados a ser claros en sus contribuciones; y finalmente el técnico, que engloba actuaciones dirigidas a ayudar a los alumnos a sentirse competentes y confortables con los recursos y las herramientas que configuran la propuesta instruccional.

A estos cuatro ámbitos, en opinión de Bravo (2012) como complemento de los anteriores debemos agregar el ámbito investigativo, ya que viene a reforzar y mantener en constante búsqueda e indagación al docente para descubrir y comprender las diversas problemáticas que pueden suscitarse tanto en el proceso de enseñanza y aprendizaje, como en su área específica de formación y conocimiento como también en el área de gestión o de la organización escolar. Pues como apunta Tobón (citado por Tobón, Rial Sánchez, Carretero y García, ob. cit), las competencias son procesos complejos de desempeño con idoneidad en determinados contextos, teniendo como base la responsabilidad y desde esta perspectiva, "la formación con base en competencias tiene como eje esencial formar no solo para la ejecución de actividades profesionales, sino también educar para aprender a analizar y resolver problemas, lo cual implica un enfoque investigativo" (p.102). En tal sentido, a continuación se presentan los 5 ámbitos que desde la perspectiva de Bravo (ob. cit) se deben valorar para el desarrollo de competencias en la e-mediación docente.

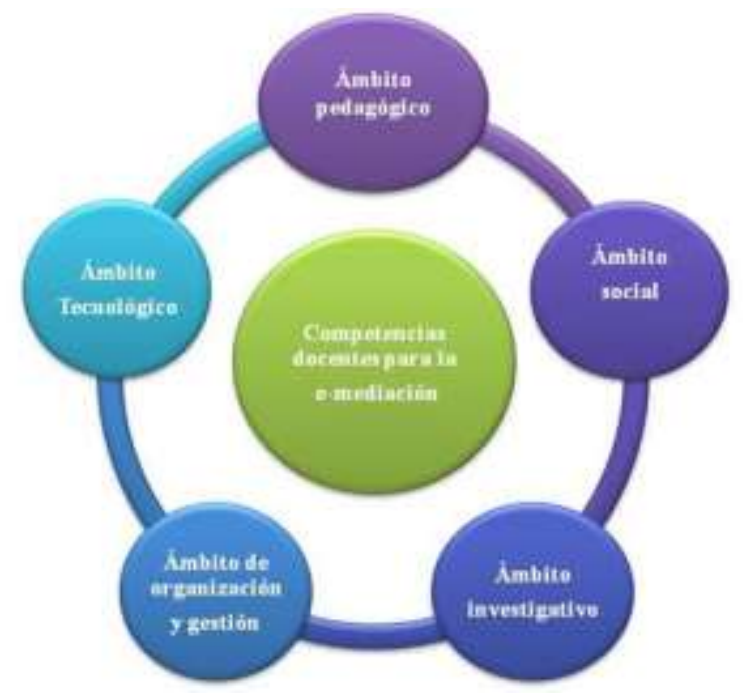

Gráfico 2. Competencias del e-mediador. Bravo (ob. cit). 


\section{Competencias TIC para la gestión del conocimiento: un aporte desde el Modelo TPACK \\ Candy Padrón y María de la Soledad Bravo ( pp. 49-73)}

\section{El pentágono de competencias TIC}

Más recientemente, el Ministerio de Educación Nacional de Colombia (2013), ha generado una interesante propuesta, en la cual se definen las competencias que deben desarrollar los docentes dentro del contexto específico de la innovación educativa con uso de TIC, para integrarlas en la mejora de los procesos de enseñanza, aprendizaje y gestión escolar. En un amplio documento, este Ministerio refiere las competencias tecnológicas, comunicativas, pedagógicas, investigativas y de gestión y las organiza en tres niveles:(a) innovador, (que implica el nivel básico); (b) integrador y (c) explorador (nivel superior).

Este trabajo constituye un referente importante y actualizado para las universidades formadoras de docentes, puesto que permite valorar competencias en los 5 ámbitos referidos y en tres niveles de profundización, además, incorpora elementos gráficos que facilitan la comprensión y posterior aplicación de la información presentada. En el gráfico 3, se muestra el pentágono de competencias y los diversos niveles desde el centro hacia la periferia. Amanera de ejemplificación se mostrará el desglose de la competencia tecnológica para evidenciar la especificidad de la información presentada, para visualizar cada una de ellas en profundidad se recomienda consultar la fuente primaria.

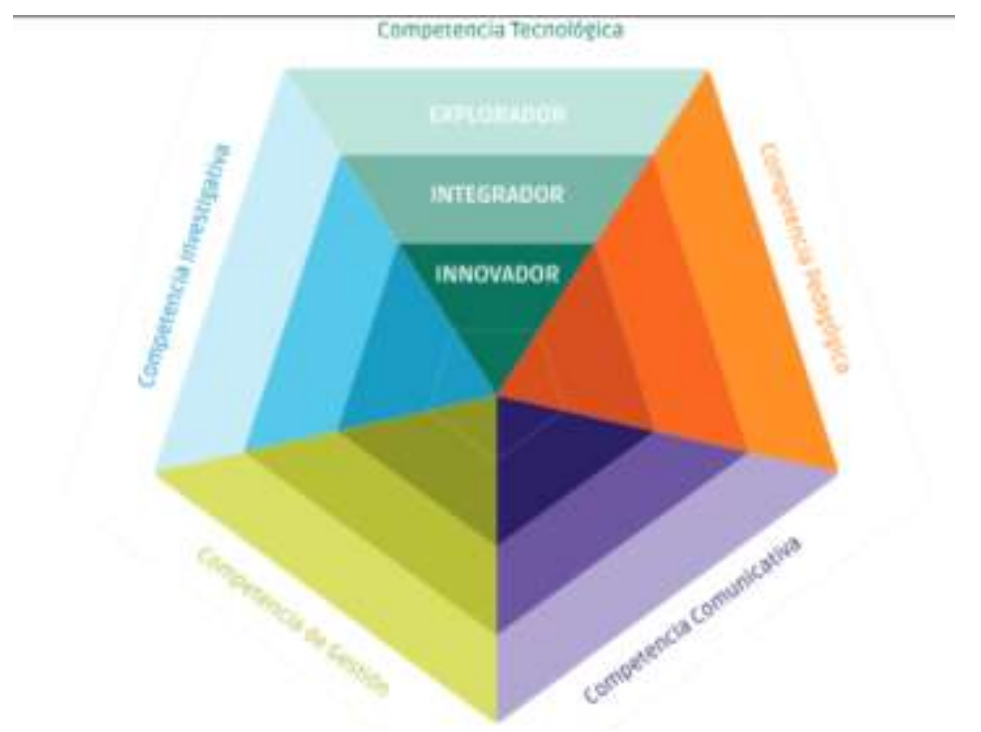

Gráfico 3: Pentágono de competencias TIC. Tomado de: Ministerio de Educación Nacional (2013). 


\section{Competencias TIC para la gestión del conocimiento: un aporte desde el Modelo TPACK}

\section{Candy Padrón y María de la Soledad Bravo ( pp. 49-73)}

La competencia tecnológica se define como la capacidad para seleccionar y utilizar de forma pertinente, responsable y eficiente una variedad de herramientas tecnológicas entendiendo los principios que las rigen, la forma de combinarlas y las licencias que las amparan. La competencia comunicativa se comprende como la Capacidad para expresarse, establecer contacto y relacionarse en espacios virtuales y audiovisuales a través de diversos medios y con el manejo de múltiples lenguajes, de manera sincrónica y asincrónica.la competencia pedagógica se constituye en el eje central de la práctica de los docentes potenciando otras competencias como la comunicativa y la tecnológica para ponerlas al servicio de los procesos de enseñanza y aprendizaje, en el ámbito de integración de las TIC, la competencia pedagógica se puede definir como la capacidad de utilizar las TIC para fortalecer los procesos de enseñanza y aprendizaje, reconociendo alcances y limitaciones de la incorporación de estas tecnologías en la formación integral de los estudiantes y en su propio desarrollo profesional.

La competencia de gestión, se puede definir como la capacidad para utilizar las TIC en la planeación, organización, administración y evaluación de manera efectiva de los procesos educativos; tanto a nivel de prácticas pedagógicas como de desarrollo institucional. Finalmente, el eje alrededor del cual gira la competencia investigativa es la gestión del conocimiento y, en última instancia, la generación de nuevos conocimientos. La investigación puede ser reflexiva al indagar por sus mismas prácticas a través de la observación y el registro sistematizado de la experiencia para autoevaluarse y proponer nuevas estrategias, por lo que se define como la capacidad de utilizar las TIC para la transformación del saber y la generación de nuevos conocimientos.

La propuesta del Ministerio colombiano plantea que las competencias se desarrollan y expresan en diferentes niveles o grados de complejidad. Así, el primer nivel o momento de exploración, se caracteriza por permitir el acercamiento a un conjunto de conocimientos que se constituyen en la posibilidad para acceder a estados de mayor elaboración conceptual. En el segundo nivel o momento de integración, se plantea el uso de los conocimientos ya apropiados para la resolución de problemas en contextos diversos. Mientras que en el tercer nivel o momento de innovación, se da mayor énfasis a los

Revista EDUCARE, Volumen 18, Número 3, Septiembre - Diciembre 2014. ISSN: 2244-7296 Página 64 


\section{Competencias TIC para la gestión del conocimiento: un aporte desde el Modelo TPACK \\ Candy Padrón y María de la Soledad Bravo ( pp. 49-73)}

ejercicios de creación; lo que permite ir más allá del conocimiento aprendido e imaginar nuevas posibilidades de acción o explicación.

Adicionalmente, se asume que cada una de las competencias del pentágono es fundamental tanto para los docentes como para los directivos de las instituciones educativas. Sin embargo, la forma en que se expresan las competencias puede variar dependiendo del momento o nivel de desarrollo en el que los docentes se encuentren, su rol, la disciplina que enseñan, el nivel en el que se desempeñan, sus intereses y sus talentos. Cada docente y directivo tiene la posibilidad de personalizar su desarrollo profesional de acuerdo a sus desempeños y características individuales, por lo que el enfoque, abre oportunidades para llevar a cabo itinerarios de desarrollo profesional situados, usando como punto de partida las necesidades e intereses de los docentes. En el gráfico 4, se muestra la competencia tecnológica en detalle, como ejemplo de lo que puede visualizarse para cada competencia y nivel propuesto.

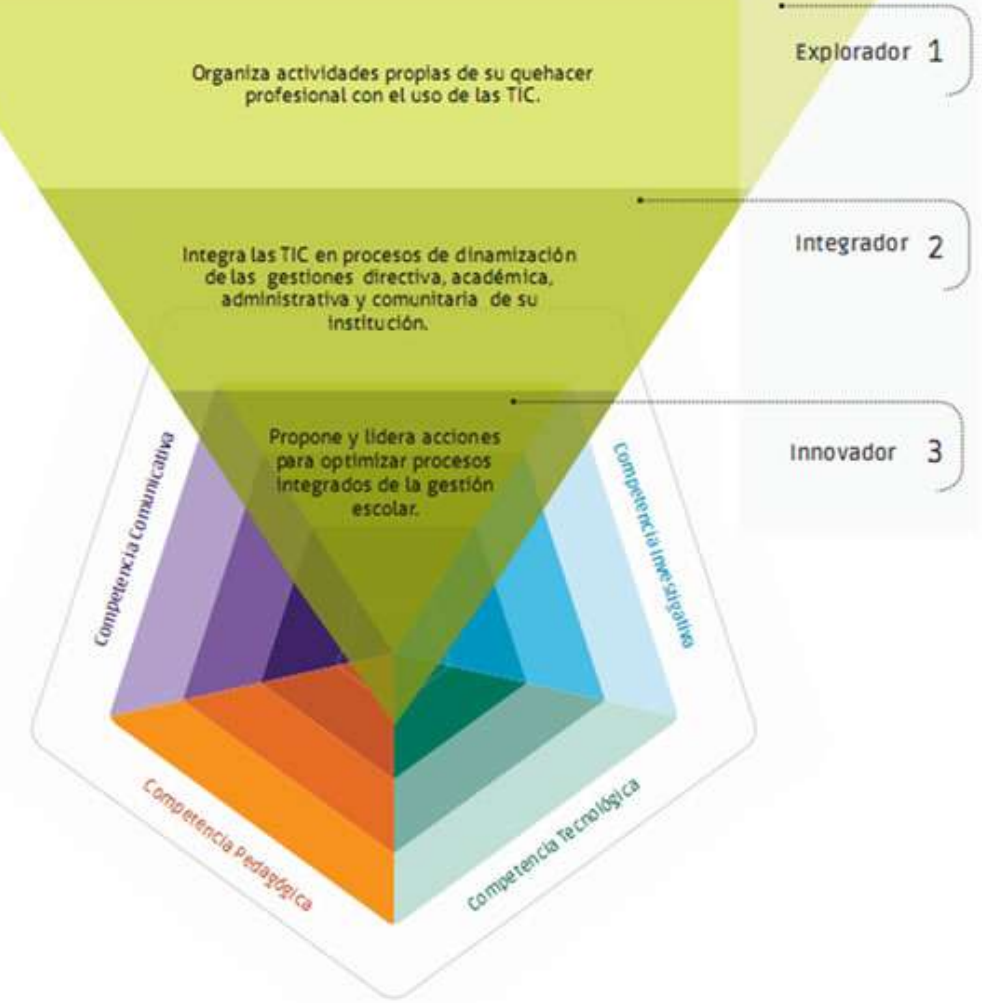

Gráfico 4. Competencia tecnológica. Tomado de: Pentágono de competencias TIC. Ministerio de Educación Nacional (ob. cit). 


\section{Competencias TIC para la gestión del conocimiento: un aporte desde el Modelo TPACK \\ Candy Padrón y María de la Soledad Bravo ( pp. 49-73)}

Se comprende que durante los primeros acercamientos de los docentes a las TIC el profesor encuentre difícil la tarea de lograr engranar estrategias didácticas, acordes al contenido y a la herramienta tecnológica más apropiada, pero una vez que lo logre, se irá familiarizando con ellasal valorar tanto la diversidad de herramientas como las bondades del uso de cada una tanto en procesos permanentes de formación como en su cotidianidad. Ciertamente, Castillo y Cabrerizo (ob. cit), afirman que:

La mejor manera de lograr esta capacitación en la utilización de las TIC como materiales curriculares es promoviendo la adecuada formación desde la propia institución en la que se desarrolla la actividad docente, incentivando su uso e integración en la tarea diaria. (p.274)

En tal sentido, se hace necesaria la implementación de modelos con los que los docentes puedan implementar progresivamente las tecnologías en los procesos didácticos, por lo que se plantea el modelo TPACK, como metodología que implique la capacitación gradual de conocimientos necesarios para la integración de las TIC.

\section{Modelo TPACK}

Cuando se trata de procesos de enseñanza y aprendizaje, lo más relevante es la planificación de los mismos para que estos se logren de la mejor manera posible. Incluso contando con los excelentes recursos, los más calificados profesores y estudiantes, las óptimas herramientas tecnológicas, entre otros, aquello que orientará dichos procesos es la planificación. En base a esto, surge un modelo de inclusión de herramientas tecnológicas a la educación, el cual toma en cuenta como elemento principal los conocimientos que manejan los docentes en las áreas de pedagogía, tecnología y el contenido que desean impartir, para realizar una planificación que incluya a las TIC. A continuación se puede observar el modelo. 


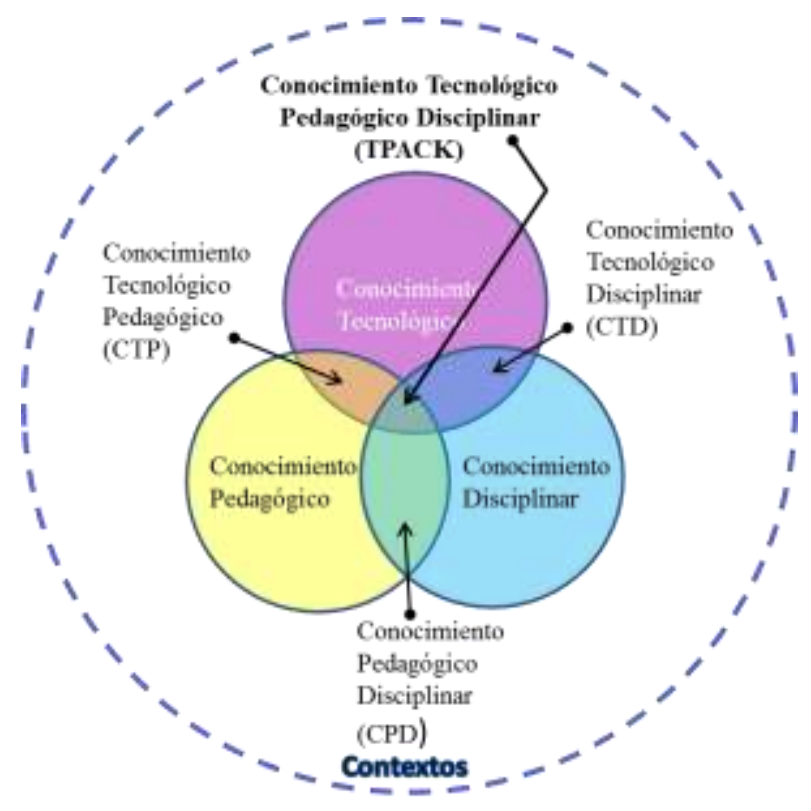

Gráfico 5. Modelo TPACK. Tomado de Mishra y Koehler, 2006.

Con respecto a la aplicación de las TIC en el aula, Mishra y Koheler (2006) plantean un modelo que recuerda que la tecnología no es el fin sino el medio para lograr los objetivos. En efecto, describen la aplicación de tres tipos de conocimientos básicos puestos en práctica en un contexto determinado, estos son conocimientos tecnológicos, pedagógicos y de contenido específico de una disciplina o materia, los cuales traducidos al inglés serían Technological, Pedagogical And Content Knowledge, (TPACK).

El modelo contempla tres tipos de conocimientos, en primer lugar, se describe el conocimiento tecnológico, el cual comprende el uso de las TIC en la cotidianidad, está relacionado al conocimiento específico de tecnologías como el internet, computación, videos. Asimismo requiere de habilidades para operar tecnologías, estas pueden ser el manejo de herramientas de software como procesadores de información, buscadores en internet, email, entre otros. Para la adquisición de dicho conocimiento basta el uso de los tutoriales presentes en muchos softwares, así como talleres básicos de inducción en el manejo de las TIC de manera general en el día a día del ser humano en la sociedad actual. 


\section{Competencias TIC para la gestión del conocimiento: un aporte desde el Modelo TPACK}

\section{Candy Padrón y María de la Soledad Bravo ( pp. 49-73)}

En segundo lugar, está el conocimiento pedagógico representado principalmente por un conocimiento sobre proceso, prácticas, teorías y métodos de enseñanza aprendizaje y como estos se relacionan para propósitos educacionales. Se debe conocer aspectos pedagógicos, manejo del ambiente de clases, planificación, ejecución y evaluación del proceso de enseñanza-aprendizaje, diseño de estrategias de enseñanza aprendizaje, conocimientos de teorías educativas así como de ciencias de la conducta. Se maneja entonces, un conocimiento que permita construir conocimiento y adquirir habilidades, bajo un entendimiento cognitivo y social aplicados a cada situación de enseñanza aprendizaje.

Por último, se identifica el conocimiento disciplinar, el cual es básicamente el conocimiento del contenido de una materia específica o una disciplina dada. Éste abarca el conocimiento del contenido que se desea enseñar, la materia y sus implicaciones curriculares. Es entonces importante tener conocimiento curricular, debido a que las diferentes asignaturas están representadas bajo un funcionamiento estructurado y diseñado por especialistas, los cuales organizan el contenido de las distintas disciplinas de manera que éste se presente de forma congruente. Las universidades pedagógicas delegan el manejo del contenido disciplinar a los departamentos y programas que manejan un contenido específico en el cual el docente saldrá especializado. De igual forma, es necesario tener conocimientos de hechos centrales, conceptos, teorías y procedimientos, entre otros, respectivos a un campo específico del contenido.

En relación a lo expuesto, pudiesen existir profesionales con estos distintos tipos de conocimiento, el tecnológico puede ser empleado en cualquier carrera universitaria, en cualquier ámbito laboral. El conocimiento pedagógico, viene dado principalmente por la formación docente, en su mayoría egresados de universidades pedagógicas. Y por último el conocimiento disciplinar, el cual es adquirido en todas las universidades, dependiendo la carrera en la cual el individuo desee egresar.

Así se puede observar, que no necesariamente todos los individuos tienen un conocimiento tecnológico, al menos tan profundo como su cotidianidad lo requiera. El conocimiento pedagógico está implicado a aquellos que quieren desarrollarse en el campo de la docencia, pero necesariamente deben ser egresados de instituciones en las que se

Revista EDUCARE, Volumen 18, Número 3, Septiembre - Diciembre 2014. ISSN: 2244-7296 Página 68 


\section{Competencias TIC para la gestión del conocimiento: un aporte desde el Modelo TPACK}

Candy Padrón y María de la Soledad Bravo ( pp. 49-73)

imparta este tipo de conocimientos, se observa entonces que no por el hecho de conocer a profundidad una disciplina, el individuo es capaz de enseñarla de la manera más apropiada, es decir, una persona puede saber hablar inglés y no por eso tiene las competencias necesarias para enseñar el idioma. Según se ha visto, hay personas que poseen solo uno de estos conocimientos o incluso dos de ellos, pero lo ideal para este modelo de integración de TIC en el aula es que el docente posea los tres conocimientos básicos para esto.

\section{Tipos de conocimientos para el empleo del modelo TPACK}

Resulta que, al entrelazar los conocimientos anteriormente señalados surgen los tres necesarios para aplicar el modelo, teniendo como objetivo principal lograr incluir las tecnologías en el proceso de enseñanza aprendizaje. Se muestra a continuación como lo que se busca es saber cómo enseñar con la tecnología, es decir, conociéndola mas no perfeccionándola.

Sobre la base del modelo TPACK, Mishra y Koheler (ob. cit.) plantean tres conocimientos que surgen de las conexiones de los mencionados anteriormente. Inician con el conocimiento Tecnológico-Pedagógico, el cual permite establecer la existencia y capacidades de componentes tecnológicos y como estos son usados para el proceso de enseñanza aprendizaje. Además, es conocer como la enseñanza puede cambiar con el resultado de usar unas tecnologías en particular. En este tipo de conocimiento, la persona debe entender que existe un rango de herramientas para tareas particulares y para la habilidad que se desea desarrollar, conociendo el diseño de estrategias pedagógicas basadas en el uso de tecnologías que incluyan herramientas como webquests, foros y sitios de chat entre otros.

Seguidamente, plantean el conocimiento Tecnológico-Disciplinar, representado por el entendimiento de que la tecnología y el contenido de una disciplina específica están recíprocamente relacionadas. Este es el conocimiento no solo del contenido, sino de cómo la materia puede estar relacionada con la aplicación de la tecnología. Finalmente, describen

el conocimiento Pedagógico-Disciplinar, el cual requiere que el individuo conozca los métodos de enseñanza que mejor se correspondan con el contenido de la materia. También,

Revista EDUCARE, Volumen 18, Número 3, Septiembre - Diciembre 2014. ISSN: 2244-7296 Página 69 


\section{Competencias TIC para la gestión del conocimiento: un aporte desde el Modelo TPACK}

\section{Candy Padrón y María de la Soledad Bravo ( pp. 49-73)}

conocer cómo los elementos del contenido pueden ordenarse de la manera más adecuada para una mejor enseñanza, existiendo la interacción del contenido con la pedagogía.

En relación a lo anterior, se nota cómo es necesario que el docente posea diversas competencias que le permitan abordar estos conocimientos con el fin de lograr una óptima introducción de las TIC en el aula. Asimismo Marqués (citado en Castillo y Cabrerizo, ob. cit), destaca como "al profesor se le reclaman competencias técnicas y pedagógicas en el uso de las tecnologías de la información y la comunicación como materiales curriculares"(p.274).

Las competencias destacadas por este autor recalcan tres tipos de conocimiento: en primer lugar "conocimiento sobre las diferentes formas de trabajar con las TIC como materiales curriculares en su ámbito docente” estableciendo así una relación entre la pedagogía y la tecnología, en segundo lugar "conocimiento organizativos y de planificación" destacando la importancia de dominar tanto la parte pedagógica como la disciplinar, y por último "Conocimientos válidos para la selección de materiales curriculares" mostrando que no solo se debe conocer la materia sino también los recursos tecnológicos más apropiados para ella, conjugando la tecnología con la disciplina. Por lo tanto, se presenta la metodología TPACK diseñado por Mishra y Koheler (ob. cit.) en la búsqueda del logro de la unión de estos tres tipos de conocimientos.

Lo planteado en el modelo TPACK, es coherente con lo que se plantea a nivel mundial sobre lo que debe ser una óptima integración de las TIC en los procesos didácticos, como lo refiere la UNESCO (ob. cit) en el proyecto NUECTID, referido anteriormente y que contempla los enfoques: nociones básicas de tecnología, profundización de los conocimientos y creación de conocimientos. La UNESCO (ob. cit.) afirma que "a través de esos enfoques, los estudiantes de un país y, en última instancia, sus ciudadanos y trabajadores adquieren competencias cada vez más sofisticadas para apoyar el desarrollo económico, social, cultural y ambiental, y la obtención de un nivel de vida mejor” (p.11). En consecuencia, es imprescindible que en todas las instituciones y fundamentalmente en las formadoras de docentes, se promueva la aplicación de modelos que ayuden a integrar las TIC en los procesos didácticos, de manera de cumplir con estos objetivos mundiales.

Revista EDUCARE, Volumen 18, Número 3, Septiembre - Diciembre 2014. ISSN: 2244-7296 Página 70 


\section{Competencias TIC para la gestión del conocimiento: un aporte desde el Modelo TPACK}

\section{Candy Padrón y María de la Soledad Bravo ( pp. 49-73)}

\section{CONCLUSIONES}

Aun cuando existen esfuerzos importantes por indicar cuáles deben ser las competencias para el desempeño eficiente del docente en el nuevo escenario educativo que implica la incorporación y uso adecuado de las TIC, todavía no se ha logrado una capitación masiva de los mismo que permita asegurar el logro de competencias generales y específicas para el uso de las TIC en diversas acciones y funciones dentro y fuera del aula, por lo tanto esta temática continua siendo de especial importancia en el ámbito educativo nacional e internacional.

Para la gestión de conocimiento en las organizaciones y sobre todo a nivel universitario, se requiere propiciar la práctica reflexiva, la cual debe incluir el aprovechamiento de todas las herramientas tecnológicas disponibles en el entorno que le permitan al docente beneficiarse en su labor cotidiana y beneficiar a sus estudiantes en la gestión del conocimiento, de modo que resulte no solo más eficiente sino también más participativa.

Considerando elementos como la práctica reflexiva y el uso de tecnologías para apoyo de la gestión de conocimiento, Azinian (ob. cit.) asegura que solo un docente capacitado y formado en la reflexión puede tomar las decisiones apropiadas para el rediseño de los entornos de aprendizaje que requiere la integración curricular de las TIC, con el fin de producir una transformación cualitativa de las prácticas pedagógicas.

Con respecto a los conocimientos establecidos en el modelo TPACK, la dimensión conocimiento Pedagógico-Disciplinar, implica que el docente sea capaz de articular de manera óptima los métodos, técnicas y estrategias pedagógicas con las asignaturas que administran. En la dimensión Conocimiento Tecnológico-Pedagógico, los docentes deben fortalecer habilidades en el manejo de equipos tecnológicos usados en clase y la variedad de herramientas tecnológicas que pueden ser utilizadas de acuerdo a la habilidad que desean desarrollar. En la dimensión Tecnológica-Disciplinar, es importante favorecerla comprensión de que la tecnología y el contenido de una disciplina específica pueden verse mutuamente beneficiadas.

Revista EDUCARE, Volumen 18, Número 3, Septiembre - Diciembre 2014. ISSN: 2244-7296 Página 71 


\section{Competencias TIC para la gestión del conocimiento: un aporte desde el Modelo TPACK}

\section{Candy Padrón y María de la Soledad Bravo ( pp. 49-73)}

Con respecto al uso de modelos de integración de las TIC, debemos reconocer, que aun cuando exista un amplio listado de las competencias TIC parta docentes, el seguir un modelo de integración, permite favorecer la implantación de las mismas a diversos niveles y de manera contextualizada con las características particulares de cada institución educativa. Lo que no podemos hacer, es esperar que el cambio se instale en la organización si no nos formamos y propiciamos el uso de las TIC desde niveles básicos de uso hasta niveles avanzados.

\section{REFERENCIAS}

Azinian, H. (2009) Las Tecnologías de la Información y la Comunicación en las Prácticas Pedagógicas Buenos Aires. Argentina: Ediciones Novedades Educativas.

Bravo, M. (2012) Investigación en TIC y docencia: temáticas, mitos y reflexiones. Trabajo presentado para el ascenso a la categoría de Profesor Titular. UPEL: IPB.

Castillo, S. y Cabrerizo, J. (2006) Formación del profesorado en educación superior. Desarrollo curricular y evaluación. Volumen II Madrid. España: Mc Graw Hill

Corredor, z. (2014) Competencias docentes en TIC en un sistema de Educación a Distancia. [Artículo en línea]. Revista de Tecnología de Información y Comunicación en $\begin{array}{llllll}\text { Educación. } & \text { Volumen } & 8, & N^{\circ} & 1 . & \text { Enero-Junio }\end{array}$ Disponible:http://servicio.bc.uc.edu.ve/educacion/eduweb/v8n1/art07.pdf. [Consultado: julio, 2014]

Díaz-Barriga Arceo, F. (2010). Los profesores ante innovaciones curriculares.[Artículo en línea]. en Revista Iberoamericana Educación Superior (RIES), México, IISUE/Universia, vol. 1, núm.1, pp. 37-57. Disponible: http://ries.universia. net/ index .php/ries/article/view/35. [Consultado: febrero, 2012]

Flores, M. y Torres, M. (2011) La Escuela como Organización del Conocimiento Mexico, D.F. Mexico : Trillas

García, L., Ruiz, M. y García, M. (2009) Claves para la educación. Actores, agentes y escenarios en la sociedad actual Madrid. España: Narcea, S. A.

Henríquez G.; Veracoechea F.; Gómez de Ugel, N. (2011). Competencias en las TIC de los docentes. educare, [S.1.], v. 14, n. 2, p. 52-74, Nov. 2011. Disponible en: http://revistas.upel.edu.ve/index.php/educare/article/view /360/168 [Consultado: Enero, 2014] 
Krüger, K. (2006) El concepto de "Sociedad del Conocimiento" [Documento en línea] Disponible en: http://www.ub.edu/geocrit/b3w-683.htm [Consultado: Septiembre 2012]

Marcelo, C., Puente, D., Ballesteros, M. y Palazón, A. (2002). Learningteleform@ción. Barcelona: Gestión 2000.com

Mauri, T. y Onrubia, J. (2008) El profesor en entornos virtuales, condiciones, perfil y competencias, en Coll, C. y Monereo, C. (Comps). Madrid: Morata.

Melaré, D. (2007) Tecnología de la inteligencia. Gestión de la competencia pedagógica virtual. Madrid. España: Editorial popular

Ministerio de Educación Nacional (2013) Competencias TIC para el desarrollo profesional docente. [Documento en línea] Disponible en: http://es.slideshare .net/ColombiaAprende/transformando-la-prctica-docente-30215610. [Consultado: Julio, 2014]

Mishra, P. y Koehler, M. (2006) Technological Pedagogical Content Knowledge: A Framework for Teacher Knowledge.Michigan StateUniversity) [Documento en línea] Disponible en: http://tpack.org/ [Consultado: Febrero 2012]

Ostériz, J. (2012, Junio 17) ¿Cosechar sin sembrar? Sistema de aprendizaje con el uso de tecnologías avanzadas (II Parte)El Impulso, p. A3.

Tobón, S., Rial, A., Carretero,M. y García,J.(2006) Competencias, calidad y educación superior. Bogotá : Magisterio.

UNESCO (2008) Normas UNESCO sobre competencias en TIC para docentes [Documento en línea] Disponible en: http://www.oei.es/tic/normas-tic-marcopoliticas.pdf[Consultado: Octubre 2012]

UNESCO (2010) El impacto de las TIC en educación. [Documento en línea] Disponible en:

unesdoc.unesco.org/images/0019/001905/190555s.pdf

[Consultado: Enero 2012] 